\title{
Decentralized Control of a Class of Large-Scale Systems by Uncertainty Estimator
}

\author{
Min-Shin Chen \\ Associate Professor, \\ Department of Mechanical Engineering, \\ National Taiwan University, \\ Taipei, Taiwan
}

A new decentralized controller is proposed for a group of subsystems subject to unknown interconnections and external disturbances. Under the assumption that the interconnections and disturbances satisfy a certain structural condition, the new controller suppresses the disturbances and intercoupling effects completely, making the overall system behave as a decoupled system. In this new control design, each local controller uses an uncertainty estimator proposed by Chen (1990) for estimation of the interconnections and disturbances, and then cancels these undesirable inputs directly. The major advantages of the proposed controller are that the interconnections need not satisfy the so-called "conical" condition, and there is no need for a priori information on the magnitudes of interconnections and disturbances.

\section{Introduction}

In controlling a large-scale system, we often use decentralized controllers due to the infeasibility of communication between subsystems or to avoid the complexity of centralized controllers. Traditional fixed-gain decentralized controllers proposed by Siljak (1978) can perform adequately in the presence of small interconnections between subsystems. However, when the size of interconnections is large, performance as well as stability can be destroyed. Later study shows that a particular class of large-scale systems, whose interconnections satisfy a certain structural condition, can always be decentralizedly stabilized even for large interconnections in the case of linear state interconnections by Sezer and Siljak (1981) and conical output interconnections by Huseyin et al. (1982). The underlying approach used by them is to synthesize each local controller to make the loop gains of the interconnected systems small so that stability can be retained in the presence of interconnections. Hence, the larger the interconnections are, the smaller the loop gains should be. A drawback of such a design approach is that small loop gains make system performance susceptible to external disturbances and system parameter variations. To remedy the situation, Gavel and Sijak (1989) suggest the use of decentralized adaptive control [4] to account for possible parameter variations. The proposed adaptive scheme can stabilize the overall system regardless of the size of interconnections when there is no external disturbance. Another approach suggested by Youcef-Toumi and Fuhlbrigge (1989) is to apply the concept of Time Delay Control. With this approach, the unknown interconnections and disturbances are directly estimated through time delay, and the control actions are modified based on the estimation results. No explicit con-

Contributed by the Dynamic Systems and Control Division for publication in the Journal of Dynamics Systems, Measurement, and Control. Manuscript received by the Dynamics and Control Division October 2, 1989. Associate Technical Editor: G. E. Young. straints are imposed on the type of interconnections; however, the interconnections and disturbances are assumed to be slowly time-varying, and evaluation of the time derivative of the system state is required in the estimation process.

In this paper we adopt an approach similar to the Time Delay Control. However, in the estimation of system interconnections and external disturbances, we use the uncertainty estimator proposed by Chen and Tomizuka (1989) and Chen (1990). As a result, we need not assume that the interconnections and disturbances are slowly time-varying. Our new controller differs from previously developed decentralized controllers in three aspects: (1) the proposed controller completely suppresses the effects of external disturbances, (2) the interconnections need not be "conical" as required by previous authors; in other words, the magnitude of the interconnections need not be bounded by the norm of the overall system state, (3) the magnitudes of interconnections and disturbances need not be known a priori. We mention that although we consider only the regulation problem, a decentralized tracking controller can be easily constructed through a slight modification of the regulation controller presented in this paper.

In the sequel, a matrix is said to be stable if all of its eigenvalues are in the open left-half plane. $\bar{\lambda}_{p}$ and $\underline{\lambda}_{p}$ denote, respectively, the maximum and minimum eigenvalues of a matrix $P$. N stands for the set $\{1,2, \ldots, N\}$, where $N>0$ is an integer, and $B_{r}$ is the set $\left\{x \in \mathbf{R}^{n}:\|x\|<r\right\}$.

\section{Problem Formulation}

Consider a system $S$, which is composed of $N$ subsystems $S_{i}, i \in \mathbf{N}$. Each subsystem is described by

$$
S_{i}: \quad \dot{x}_{i}=A_{i} x_{i}+B_{i} u_{i}+P_{i} w_{i}+Q_{i} d_{i}
$$

where $x_{i} \in \mathbf{R}^{n_{i}}$ and $u_{i} \in \mathbf{R}$ are the state and input of $S_{i}, d_{i} \in \mathbf{R}^{h_{i}}$ is 
an external disturbance, and $w_{i} \in \mathbf{R}^{m_{i}}$ is the unknown interconnections between $S_{i}$ and other subsystems, which is of the form

$$
w_{i}=f_{i}\left(x_{1}, x_{2}, \ldots, x_{N}\right)
$$

The overall system is then described by

$$
S: \quad \dot{x}=A x+B u+P w+Q d
$$

where

$$
\begin{aligned}
& A=\operatorname{diag}\left\{A_{1}, A_{2}, \ldots, A_{N}\right\} \\
& B=\operatorname{diag}\left\{B_{1}, B_{2}, \ldots, B_{N}\right\} \\
& P=\operatorname{diag}\left\{P_{1}, P_{2}, \ldots, P_{N}\right\} \\
& Q=\operatorname{diag}\left\{Q_{1}, Q_{2}, \ldots, Q_{N}\right\}
\end{aligned}
$$

$x=\left(x_{1}^{T}, x_{2}^{T}, \ldots, x_{N}^{T}\right)^{T}, u=\left(u_{1}^{T}, u_{2}^{T}, \ldots, u_{N}^{T}\right)^{T}, w=\left(w_{1}^{T}, w_{2}^{T}, \ldots, w_{N}^{T}\right)^{T}$ and $d=\left(d_{1}^{T}, d_{2}^{T}, \ldots, d_{N}^{T}\right)^{T}$.

The objective is to develop a decentralized controller for each subsystem $S_{i}$, which achieves regulation of the system state $x_{i}$ in the presence of the external disturbance $d_{i}$ and the unknown interconnection $w_{i}$. We make the following assumptions on every subsystem:

(A1) $A_{i}$ is a stable matrix. (If $A_{i}$ is not a stable matrix, we can always use state feedback control to stabilize the uninterconnected system by assuming the controllability of $\left(A_{i}, B_{i}\right)$.)

(A2) There exist $p_{i} \in \mathbf{R}^{1 \times m_{i}}$ and $q_{i} \in \mathbf{R}^{1 \times h_{i}}$ such that

$$
P_{i}=B_{i} p_{i} \text { and } Q_{i}=B_{i} q_{i}
$$

(A3) The external disturbance $d_{i}$ satisfies

$$
\left|d_{i}(t)\right|<D_{i 0} \text { and } / \dot{d}_{i}(t) /<D_{i 1} \text { for all } t>0
$$

(A4) The interconnection $f_{i}(x)$ in Eq. (2) is such that for any $r_{0}>0$, there exists a finite time $T\left(r_{0}\right)>0$ so that given any disturbances specified in (A3), if $x(0)=x_{0} \in B_{r}$, the solution of the open-loop system $x(t)$ can grow at most exponentially with exponent $\zeta>0$ for all $t \in[0, T]$. Hence,

$$
\text { if } x_{0} \in B_{r 0} \Rightarrow x(t) \in B_{r} \subset B_{r_{p}} \forall t \in[0, T]
$$

where $r=r_{0} e^{5 T}, r_{p}=\left(\bar{\lambda}_{p} / \underline{\lambda}_{p}\right)^{1 / 2} r+\kappa$ with $\kappa$ a small positive number, and $P$ is the solution of the Lyapunov Equation

$$
A^{T} P+P A=-I
$$

with the matrix $A$ as given in Eq. (3). In particular, we assume that $f_{i}$ and its first derivative are continuous; hence, we have the following upper bounds:

$$
\begin{array}{ll}
\left\|f_{i}\right\|<L_{r_{p}} & \forall x \in B_{r_{p}} \\
\left\|\partial f_{i} / \partial x\right\|<M_{r_{p}} & \forall x \in B_{r_{p}}
\end{array}
$$

Combining the structural condition (A2) with Eq. (1), we can rewrite the subsystem dynamics as

$$
S_{i}: \quad \dot{x}_{i}=A_{i} x_{i}+B_{i}\left(u_{i}+e_{i}\right)
$$

where $e_{i}=p_{i} w_{i}+q_{i} d_{i}$ is an equivalent uncertainty viewed from the input channel. Similarly, Eq. (3) is rewritten as

$$
S: \quad \dot{x}=A x+B(u+e)
$$

where $e=\left(e_{1}^{T}, \mathrm{e}_{2}^{T}, \ldots, e_{N}^{T}\right)^{T}$.

Using Assumptions (A1)-(A4) and Eq. (7) with $u=0$, we show in Appendix $A$ that if $x_{0} \in B_{r 0}$, the equivalent uncertainty $e_{i}$, for all $i \in N$, satisfies

$$
\left|\dot{e}_{i}\right| \leq \eta_{i 1}\left|e_{i}\right|+\eta_{i 0}, \quad \forall x \in B_{r_{p}}
$$

for some positive constants $\eta_{i 1}$ and $\eta_{i 0}$. Eq. (8) implies that if $x_{0} \in B_{r 0}$ and there is no control $\left(u_{i}=0\right)$, the equivalent uncertainty $e_{i}$ can grow at most exponentially with exponent $\eta_{i 1}$ before $x$ exits the ball $B_{r_{p}}$.

\section{Uncertainty Estimator}

We now follow the procedure developed by Chen (1990) to set up an uncertainty estimator for on-line estimation of the equivalent uncertainty $e_{i}$ in Eq. (6). For each subsystem $S_{i}$, define an output

$$
y_{i}=C_{i} x_{i} \text { with } C_{i} B_{i}=1
$$

Taking the time derivative of $y_{i}$ yields

$$
\dot{y}_{i}=C_{i} A_{i} x_{i}+u_{i}+e_{i}
$$

Before estimating $e_{i}$, we need first obtain an upper bound of its magnitude. Construct the following uncertainty-bound estimator based on Eq. (10):

$$
\begin{aligned}
& \dot{z}_{i 1}=C_{i} A_{i} x_{i}+u_{i}+\left(\rho_{i}-\lambda_{0}\right) / \lambda_{1} \\
& \dot{z}_{i 2}=C_{i} A_{i} x_{i}+u_{i}-\left(\rho_{i}-\lambda_{0}\right) / \lambda_{1}
\end{aligned}
$$

where $\lambda_{1}$ and $\lambda_{0}$ are two positive constants with $\lambda_{1}>1$, and $\rho_{i}$, which is an estimate of a magnitude upper bound of $e_{i}$, is governed by

$$
\begin{aligned}
\dot{\rho}_{i} & =\nu_{i}\left(\mu_{1} \rho_{i}+\mu_{0}\right), \quad \rho_{i}(0) \geq \lambda_{0} \\
\nu_{i} & = \begin{cases}1 & \dot{\epsilon}_{i 1} \dot{\epsilon}_{i 2}>0 \\
0 & \text { otherwise }\end{cases}
\end{aligned}
$$

with $\epsilon_{i 1}=y_{i}-z_{i 1}, \epsilon_{i 2}=y_{i}-z_{i 2}$, and $\mu_{1}$ and $\mu_{0}$ two positive constants.

Lemma 1: If the uncertainty-bound estimator (11) is applied to the free system (1) $\left(u_{i}=0\right.$ for all $\left.i \in N\right)$ subject to Assumptions (A1)-(A4), given any $T_{0}>0$, there exist sufficiently large $\mu_{1}$ and $\mu_{0}$ in Eq. (11c) such that

$$
\rho_{i}(t) \geq \lambda_{1}\left|e_{i}\right|_{t}+\lambda_{0} \geq \lambda_{1}\left|e_{i}(t)\right|+\lambda_{0} \text { for all } t \geq \mathrm{T}_{0}
$$

where $\left|e_{i}\right|_{t} \equiv \sup _{T_{0} \leq \tau \leq t}\left|e_{i}(\tau)\right|$.

Proof: See Appendix B.

Remark: To avoid $z_{i 1}$ and $z_{i 2}$ blowing up to infinity, we can reset them to zero when their magnitudes exceed a certain prescribed bound. This reset action will not affect the result of Lemma 1.

Finally, we construct the following uncertainty estimator:

$$
\begin{aligned}
\dot{\chi}_{i} & =C_{i} A_{i} x_{i}+u_{i}+\nu_{i} \\
\nu_{i} & =\sigma s_{i}+\rho_{i} g\left(s_{i}\right) \\
s_{i} & =y_{i}-\chi_{i}
\end{aligned}
$$

where $\sigma$ is an arbitrary positive constant, $\rho_{i}$ is given by the uncertainty-bound estimator $(11), g(\bullet) \in C^{l}$ can be any monotonically increasing odd function ranging from -1 to +1 . The variable $v_{i}$ in Eq. $(12 b)$ then becomes an estimate of the uncertainty $e_{i}$ in Eq. (10). This result is shown in Lemma 2.

Lemma 2: If the uncertainty estimator (12) is applied to the free system (1) $\left(u_{i}=0\right.$ for all $\left.i \in N\right)$ subject to Assumptions (A1)-(A4), we obtain the following results:

(I) There exists a finite time $T_{1}\left(>T_{0}\right.$, where $T_{0}$ is as given in Lemma 1) such that

$$
s_{i}(t) \in M_{i 1}, \quad \forall t \geq T_{\mathrm{i}}
$$

where $M_{i 1}=\left\{s_{i}:\left|s_{i}\right| \leq r_{i 1}, r_{i 1}=g^{-1}\left(1 / \lambda_{1}+\gamma_{i}\right), \gamma_{1}>0\right.$ is an arbitrarily small number satisfying $\gamma_{1}<1-1 / \lambda_{1}$ \}

(II) There exists a finite time $T_{2}\left(>T_{1}\right)$ such that

$$
\dot{s}_{i}(t)\left(=\nu_{i}-e_{i}\right) \in M_{i 2}, \quad \forall t \geq T_{2}
$$

where $\quad M_{i 2}=\left\{\dot{s}_{i}:\left|\dot{s}_{i}\right| \leq r_{i 2}, r_{i 2}=\mu_{1} \frac{\pi_{i 1}}{\pi_{i 2}}+\max \left(\frac{\eta_{1}}{\pi_{i 2} \lambda_{1}}, \frac{\eta_{0}}{\pi_{i 2} \lambda_{0}}+\right.\right.$

$\left.\frac{\mu_{0} \pi_{i 1}}{\lambda_{0} \pi_{i 2}}\right)+\gamma_{2}, \pi_{i 1}=\sup _{s_{i} \in M_{i 1}} / g\left(s_{i}\right) /, \pi_{i 2}=\inf _{s_{i} \in M_{i 1}} g^{\prime}\left(s_{i}\right)$ and $\gamma_{2}>0$ is an arbitrarily small number 
(III) $T_{1}-T_{0}$ and $T_{2}-T_{1}$ can be made arbitrarily small if we use sufficiently large $\sigma$ in Eq. (12b), and $\lambda_{1}, \lambda_{0}$ in Eq. (11).

Proof: See Appendix C.

Remark: It is clear from the definition of $M_{i 1}$ that we can choose $\lambda_{1}$ in Eq. (11) sufficiently large so that $r_{i 1}$ is arbitrarily small. Consequently, $r_{i 2}$ in $M_{i 2}$ is also small if $\lambda_{1}$ and $\lambda_{0}$ are large enough since $\pi_{i 1} / \pi_{i 2}$ is in the order of $r_{i 1}$. For example,

$$
\frac{\pi_{i 1}}{\pi_{i 2}}=\frac{r_{i 1}\left(r_{i 1}+\delta\right)}{\delta} \text { when } g(s)=\frac{s}{|s|+\delta}
$$

Hence, Part II of Lemma 2 implies that given an arbitrarily small $r_{i 2}$ there always exist large enough $\sigma, \lambda_{1}$ and $\lambda_{0}$ such that the estimation error $\left|e_{i}-\nu_{i}\right|$ becomes bounded by $r_{i 2}$.

\section{Decentralized Controller}

Having obtained an estimate of the equivalent uncertainty $e_{i}$, the decentralized control law is given by

$$
u_{i}=-\nu_{i} \text { for all } t \geq T_{2}
$$

where $\nu_{i}$ is given by the uncertainty estimator (12), and $T_{2}$ is as given in Lemma 2.

Theorem: Consider the system (1) subject to Assumptions (A1)-(A4). Given any initial condition $x_{0} \in B_{r 0}$, if the parameters $\mu_{1}, \mu_{0}, \lambda_{1}$, and $\lambda_{0}$ in the uncertainty-bound estimator (11) and $\sigma$ in the uncertainty estimator (12) are chosen sufficiently large so that $T_{2}<T$, where $T$ is as given in Assumption (4), and $T_{2}$ as in Lemma 2 , and the control law (13) is applied to the system (1), the overall system state $x$ converges to a small neighborhood of the origin in the state space, and all the signals in the controller remain bounded.

\section{Proof: See Appendix D.}

Remark: The control law (13) stipulates that we know $T_{2}$; that is, we know when the estimator (12) achieves estimation of $e_{i}$. Unfortunately, we have only access to the signal $e_{i}$, implying that we can determine only $T_{1}$ instead of $T_{2}$. However, since Lemma 2 says that we can choose $\lambda_{1}, \lambda_{0}$ in Eq. (11) and $\sigma$ in Eq. $(12 b)$ sufficiently large so that $T_{2}$ is very close to $T_{1}$, in real implementation we can switch on the control law (13) at $t=T_{1}$. Actually, experience gained from simulation studies shows that $T_{2}$ coincides almost with $T_{1}$ even for small values of $\lambda_{1}, \lambda_{0}$, and $\sigma$.

Example: A four-dimensional system is simulated to verify the proposed decentralized control law.

$$
\begin{array}{ll}
S_{1}: & \dot{x}_{1}=x_{2} \\
& \dot{x}_{2}=-24 x_{1}-10 x_{2}+u_{1}+w_{1}+d_{1} \\
S_{2}: & \dot{x}_{3}=x_{4} \\
& \dot{x}_{4}=-6 x_{3}-4 x_{4}+u_{2}+w_{2}+d_{2}
\end{array}
$$

where

$$
\begin{array}{ll}
w_{1}=20 x_{1} x_{4}, & d_{1}=\sin (t) \\
w_{2}=5 x_{2} x_{3}, & d_{2}=20
\end{array}
$$

Figure 1 shows performance of the uncontrolled system, where the magnitudes of $x_{1}$ and $x_{3}$ grow in an almost linear manner. Actually, all the state variables explode to infinity at about 3.8 seconds. We apply the control law (13) with the following parameters

$$
\begin{array}{lll}
\lambda_{1}=10 & \lambda_{0}=6.25 & \sigma=1 \\
\mu_{1}=10 & \mu_{0}=10 & g(s)=s /(/ s /+1)
\end{array}
$$

In implementation of Eq. (11d), we use the approximation

$$
\operatorname{sign}\left(\dot{\epsilon}_{i 1}(t)\right) \approx \operatorname{sign}\left(\epsilon_{i 1}(t)-\epsilon_{i 1}(t-\Delta)\right)
$$

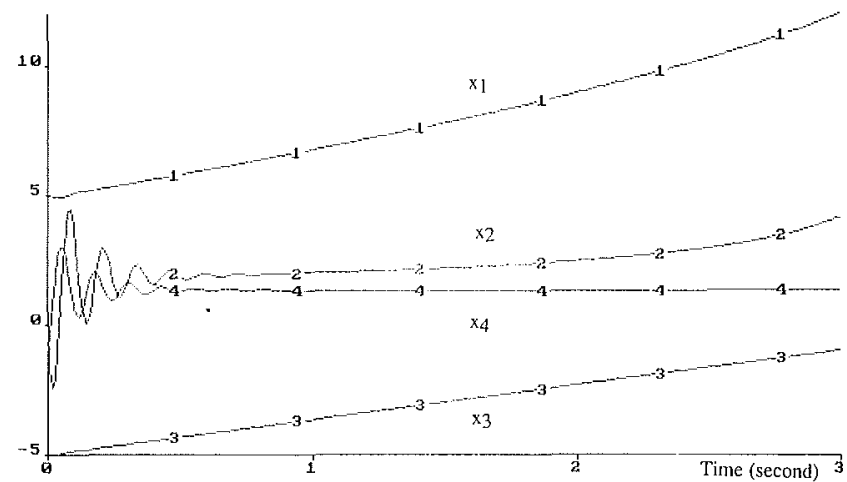

Fig. 1 Open-loop state response of the interconnected system

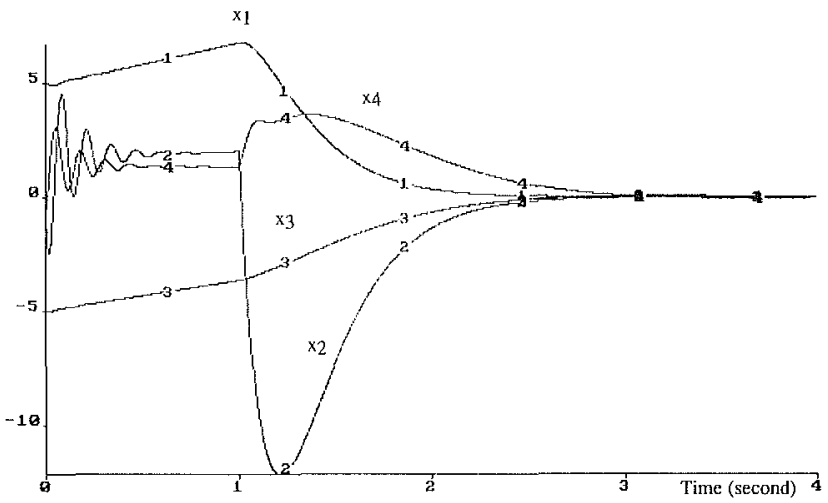

Fig. 2 State response of the Decentralized System

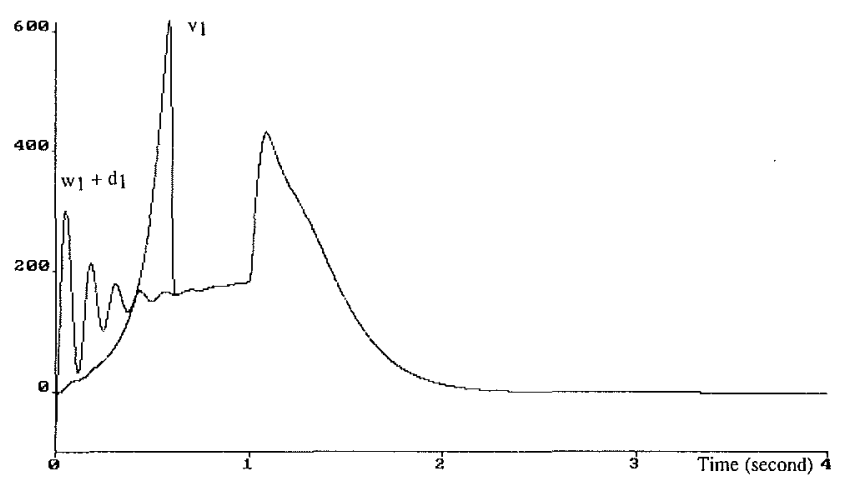

Fig. 3 Uncertainty $w_{1}+d_{1}$ and estimated uncertainty $v_{1}$

to determine the sign of $\epsilon_{i 1}$, where $\Delta=0.01$ second, and $\operatorname{sign}\left(\dot{\epsilon}_{i 2}(t)\right)$ is determined similarly. The control law is switched on at $t=1$ second although estimation of the uncertainties is achieved at about 0.7 second. The convergence of the system state to zero is verified by Fig. 2. Figures 3 and 4 show the estimates of uncertainties $w_{1}+d_{1}$ and $w_{2}+d_{2}$, respectively.

\section{Conclusions}

We apply the uncertainty estimator proposed by Chen (1990) to synthesize a decentralized controller for a group of subsystems subject to unknown interconnections and external disturbances. The new controller estimates and cancels the unknown interconnections and the disturbances so that the overall system behaves as a decoupled system with no external disturbances. The advantages of the new controller are that the interconnections may be a nonlinear function of the system state (not necessarily satisfying the conical condition), and the 


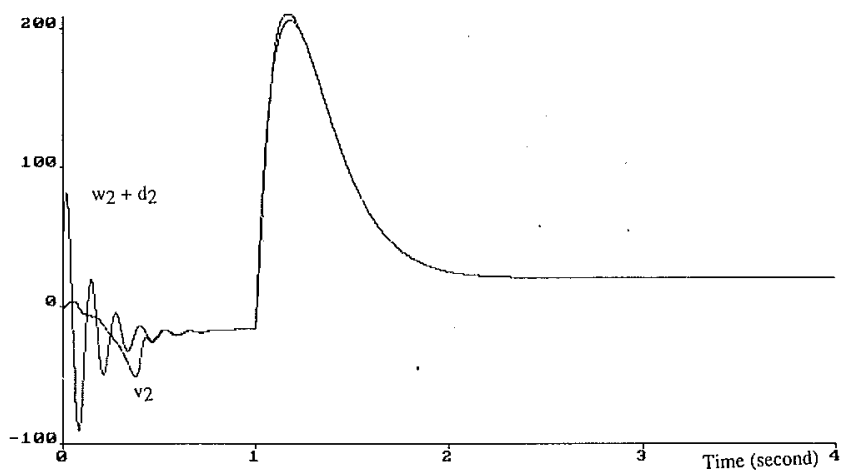

Fig. 4 Uncertainty $w_{2}+d_{2}$ and estimated uncertainty $v_{1}$

magnitudes of the interconnections and disturbances can be of any bounded unknown size.

In this paper we proved the existence of large enough controller parameters which ensure the convergence of the system state; however, we did not explicitly show, given an initial condition, what the minimum values of these controller parameters are. One possible solution to this problem is to devise an adaptive version of the uncertainty (-bound) estimator. Nevertheless, we mention that overly large parameters in the uncertainty (-bound) estimators will not deteriorate the system performance in our case since large parameters affect the uncertainty estimate only during the transient period of the estimation process. Once the estimate has successfully tracked the uncertainty, the magnitudes of those parameters are no longer reflected in the estimation results. Since our control is not switched on until the uncertainty estimation is achieved, we do not need to worry about the system performance when using large parameters in the estimators. However, we have to mention that the above comment is true only when the measurement noise is negligible. When there is significant measurement noise, the problem of using large parameters in the proposed uncertainty estimator must be considered due to the weak robustness of the estimator to stochastic noise. How to make the estimator immune to the measurement noise is now under investigation.

\section{References}

Chen, M. S., 1990, "Estimation of System Uncertainties," Proc. of the 1990 American Control Conference, San Diego, Calif., pp. 2020-2024.

Chen, M. S., and Tomizuka, M., 1989, "Disturbance Estimator and Its Application in Estimation of System Output Derivatives," Proc. of the 28th IEEE Conference on Decision and Control, Tampa, Fla., pp. 452-457.

Gavel, D. T., and Siljak, D. D., 1989, "Decentralized Adaptive Control: Structural Conditions for Stability," IEEE Trans., A.C., Vol. 34, Apr., pp. 413-426.

Huseyin, O., Sezer, M. E., and Siljak, D. D., 1982, "Robust Decentralized Control Using Output Feedback," IEE Proc., Vol. 129-D, No. 6, pp. 310-314.

Narendra, K. S., Annaswamy, A. M., and Singh, R. P., 1985, "A General Approach to the Stability Analysis of Adaptive Systems," Int. J. Contr., Vol. 41, pp. 193-216.

Sezer, M. E., and Siljak, D. D., 1981, "On Decentralized Stabilization and Structure of Linear Large-Scale Systems," Automatica, Vol. 17, pp.641-644.

Siljak, D. D., 1978, Large-Scale Dynamic Systems: Stability and Structure, North-Holland, New York.

Youcef-Toumi, K., and Fuhlbrigge, T. A., 1989, "Application of a Decentralized Time Delay Controller to Robot Manipulators," Proc. of the Robotic Conference, pp. 1786-1791.

\section{A P P E N D I X A}

Derivation of Eq. (8): From the definition of $e_{i}$ and Assumptions (A3) and (A4), we obtain

$$
\begin{aligned}
\left|e_{i}\right| & \leq \pi_{i}\left\|w_{i}\right\|+\theta_{i}\left\|d_{i}\right\| \\
& \leq \pi_{i} L_{r_{p}}+\theta_{i} D_{i 0} \equiv e_{i} \quad \forall x \in B_{r_{p}}
\end{aligned}
$$

where $\pi_{i}=\left\|p_{i}\right\|, \theta_{i}=\left\|q_{i}\right\|$, and $L_{r_{p}}, D_{i 0}$ are as given, respectively, in Assumptions (A3) and (A4).

Applying Lemma 3 in Narendra et al. (1985) to Eq. (7) with $u=0$ yields

$$
\|x(t)\| \leq C_{i}\|e\|_{t}+C_{2} \text {, where } C_{1}, C_{2} \in \mathbf{R}^{+}
$$

Taking the norm of Eq. (7), and using (A.2), we obtain

$$
\|\dot{x}(t)\| \leq C_{3}\|e\|_{t}+C_{4}
$$

where $C_{3}=\|A\| C_{1}+\|B\|$ and $C_{4}=\|A\| C_{2}$. We now take the derivative of $w_{i}$ :

$$
\begin{aligned}
\left\|\dot{w}_{i}\right\| & \leq\left\|\partial f_{i} / \partial x\right\|\|\dot{x}\| \\
& \leq M_{r_{p}} C_{3}\|e\|_{t}+M_{r_{p}} C_{4} \quad \forall x \in B_{r_{p}}
\end{aligned}
$$

Hence,

$$
\begin{aligned}
\left|\dot{e}_{i}\right| & =\left|p_{i} \dot{w}_{i}+q_{i} \dot{d}_{i}\right| \\
& \leq \pi_{i}\left\|\dot{w}_{i}\right\|+\theta_{i} D_{i 1} \\
& \leq \pi_{i} M_{r_{p}} C_{3}\|e\|_{\ell}+\pi_{i} M_{r_{p}} C_{4}+\theta_{i} D_{i 1} \quad \forall x \in B_{r_{p}}
\end{aligned}
$$

Finally, noting that $\|e\|_{t} \leq \sum_{i}\left|e_{i}\right|_{t}$, and using (A.1), we obtain

$$
\left|\dot{e}_{i}\right| \leq \eta_{i 1}\left|e_{i}\right|+\eta_{i 0} \quad \forall x \in B_{r_{p}}
$$

where $\eta_{i 1}=\pi_{i} M_{r_{p}} C_{3}$ and $\eta_{i 0}=\pi_{i} M_{r_{p}} C_{3} \sum_{j \neq i} e_{j}+\pi_{i} M_{r_{p}} C_{4}+\theta_{i} D_{i 1}$.

\section{A P P E N D I X B}

Proof of Lemma 1: From Eq. (8), we know that $e_{i}(t)$ can grow at most exponentially. In fact, we have

$$
\left|e_{i}(t)\right| \leq\left(\left|e_{i}(0)\right|+\eta_{i 0} / \eta_{i 1}\right) e^{\eta_{i 1} t} \quad \forall x \in B_{r_{p}}
$$

Subtracting Eqs. (11a) and (11b) from Eq. (10), respectively, yields

$$
\dot{\epsilon}_{i 1}=-\left(\rho_{i}-\lambda_{0}\right) / \lambda_{1}+e_{i} \text { and } \dot{\epsilon}_{i 2}=\left(\rho_{i}-\lambda_{0}\right) / \lambda_{1}+e_{i}
$$

$\dot{\epsilon}_{i 1} \dot{\epsilon}_{i 2}>0$ implies that $\rho_{i}(t)<\lambda_{1}\left|e_{i}(t)\right|+\lambda_{0}$; thus, $\rho_{i}(t)$ grows exponentially with exponent $\mu_{1}$ by the choice of $\nu$ in Eq. (11d). If we choose $\mu_{i 1}>\eta_{i 1}$, given any initial conditions $e_{i}(0)$ and $\rho_{i}(0)>\lambda_{0}$, there exists a finite time $T_{0}$ such that $\rho_{i}(t) \geq \lambda_{1}\left|e_{i}(t)\right|+\lambda_{0}$ for all $t \geq T_{0}$. Since $\rho_{i}(t)$ is a monotonically increasing function of time, we have

$$
\rho_{i}(t) \geq \lambda_{1}\left|e_{i}\right|_{t}+\lambda_{0} \text { for all } t \geq T_{0} .
$$

where $\left|e_{i}\right|_{t} \equiv \sup _{T_{0} \leq \tau \leq t}\left|e_{i}(\tau)\right|$.

\section{A P P E N D I X C}

Proof of Lemma 2: Part (I) Define $V_{1}=1 / 2 s_{i}^{2}$. Let $T_{0}$ be as given in Lemma 1 , then

$$
\begin{aligned}
\dot{V}_{1} & =s_{i}\left(-\sigma s_{i}-\rho_{i} g\left(s_{i}\right)+e_{i}\right) \quad \forall t \geq T_{0} . \\
& \leq-\sigma s_{i}^{2}-\rho_{i}\left|s_{i}\right| g\left(\left|s_{i}\right|\right)+\left|s_{i}\right|\left|e_{i}\right| \\
& \leq-\sigma s_{i}^{2}-\left(\lambda_{1}\left|e_{i}\right|+\lambda_{0}\right)\left|s_{i}\right|\left(g\left(\left|s_{i}\right|\right)-\left|e_{i}\right| /\left(\lambda_{1}\left|e_{i}\right|+\lambda_{0}\right)\right) \\
& \leq-\sigma s_{i}^{2}-\left(\lambda_{1}\left|e_{i}\right|+\lambda_{0}\right)\left|s_{i}\right|\left(g\left(\left|s_{i}\right|\right)-1 / \lambda_{1}\right) \\
& \leq-\sigma r_{i 1}^{2}-\left(\lambda_{1}\left|e_{i}\right|+\lambda_{0}\right) r_{i 1} \gamma_{1} \quad \forall s_{i} \notin M_{i 1}
\end{aligned}
$$

where we have used the result of Lemma 1 in the second inequality. Since $V_{1}(t) \geq 0$, we conclude that $s_{i}$ must enter $M_{i 1}$ within a finite period of time, say $T_{1}$, and stays inside $M_{i 1}$ thereafter. Furthermore, from Eq. (C.1) we know that $T_{1}-T_{0}$ can be made arbitrarily small if $\lambda_{1}, \lambda_{0}$ and $\sigma$ are chosen sufficiently large. 
Part (II) Define $V_{2}=1 / 2 \dot{s}_{i}^{2}$. Then

$$
\begin{aligned}
\dot{V}_{2}=\dot{s}_{i}\left(-\sigma \dot{s}_{i}-\nu \mu_{1} \rho_{i} g\left(s_{i}\right)-\nu \mu_{0} g\left(s_{i}\right)-\rho_{i} g^{\prime}\left(s_{i}\right) \dot{s}_{i}+\dot{e}_{i}\right) \\
\leq-\sigma \dot{s}_{i}^{2}-\rho_{i} \dot{s}_{i}^{2} g^{\prime}\left(s_{i}\right)+\left|\dot{s}_{i}\right| \mu_{1} \rho_{i}\left|g\left(s_{i}\right)\right| \\
+\left|\dot{s}_{i}\right| \mu_{0}\left|g\left(s_{i}\right)\right|+\left|\dot{s}_{i}\right|\left|\dot{e}_{i}\right| \\
\leq-\sigma \dot{s}_{i}^{2}-\rho_{i} \dot{s}_{i}^{2} g^{\prime}\left(s_{i}\right)+\left|\dot{s}_{i}\right| \mu_{1} \rho_{i}\left|g\left(s_{i}\right)\right| \\
+\left|\dot{s}_{i}\right| \mu_{0}\left|g\left(s_{i}\right)\right|+\left|\dot{s}_{i}\right|\left(\eta_{1}\left|\dot{e}_{i}\right|+\eta_{0}\right)
\end{aligned}
$$

Since $s_{i}(t) \in M_{i 1}$ after $t \geq T_{1}$, defining $\pi_{i 1}=\sup _{s_{i} \in M_{i 1}}\left|g\left(s_{i}\right)\right|$ and $\pi_{i 2}=\inf _{s_{i} \in M_{i 1}} g^{\prime}\left(s_{i}\right)$, we obtain

$$
\begin{gathered}
\dot{V}_{2} \leq-\sigma \dot{s}_{i}^{2}-\rho_{i} \dot{s}_{i}^{2} \pi_{i 2}+\left|\dot{s}_{i}\right| \mu_{1} \rho_{i} \pi_{i 1}+\left|\dot{s}_{i}\right| \mu_{0} \pi_{i 1} \\
+\eta_{1}\left|\dot{s}_{i}\right|\left|e_{i}\right|_{t}+\eta_{0}\left|\dot{s}_{i}\right| \quad \forall t \geq T_{1} \\
\times\left(\left|\dot{s}_{i}\right|-\mu_{1} \frac{\pi_{i 1}}{\pi_{i 2}}-\frac{\eta_{1}\left|e_{i}\right|_{t}+\eta_{0}+\mu_{0} \pi_{i 1}}{\pi_{i 2} \lambda_{1}\left|e_{i}\right|_{t}+\pi_{i 2} \lambda_{0}}\right) \\
\leq-\sigma \dot{s}_{i}^{2}-\left(\lambda_{1}\left|e_{i}\right|_{t}+\lambda_{0}\right)\left|\dot{s}_{i}\right| \pi_{i 2} \\
\times\left(\left|\dot{s}_{i}\right|-\mu_{1} \frac{\pi_{i 1}}{\pi_{i 2}}-\max \left(\frac{\eta_{1}}{\pi_{i 2} \lambda_{1}}, \frac{\eta_{0}}{\pi_{i 2} \lambda_{0}}+\frac{\mu_{0} \pi_{i 1}}{\lambda_{0} \pi_{i 2}}\right)\right) \\
\leq-\sigma r_{i 2}^{2}-\left(\lambda_{1}\left|e_{i}\right|_{t}+\lambda_{0}\right) r_{i 2} \pi_{i 2} \gamma_{2} \quad \forall \dot{s}_{i} \notin M_{i 2}
\end{gathered}
$$

where we have used the result of Lemma 1 again in the second inequality. From the last inequality we conclude that $\dot{s}_{i}$ must enter $M_{i 2}$ within a finite period of time, say $T_{2}$, and stays inside $M_{i 2}$ thereafter. Furthermore, from Eq. (C.2) we know that $T_{2}-T_{1}$ can be made arbitrarily small if $\lambda_{1}, \lambda_{0}$, and $\sigma$ are chosen sufficiently large.

\section{A P P E N D I X D}

Proof of the Theorem. We first make an observation that if Eq. (8) remains true for the same parameters $\eta_{i 1}$ and $\eta_{i 0}$ before and after $t=T_{2}$, we retain the results of Lemma 1 and Lemma 2 for all $t \geq T_{2}$. These results then justify the Theorem. Hence, to prove the Theorem, all we need to show is that Eq. (8) remains true after $t=T_{2}$. A careful examination of the derivation of Eq. (8) in Appendix A shows that in fact all we need to do is to verify that under the condition $\left|u_{i}+e_{i}\right| \leq r_{i 2}$, $x(t)$ remains in $B_{r_{p}}$ for all $t \geq T_{2}$.

If $\left|u_{i}+e_{i}\right| \leq r_{i 2}$ for all $i \in N$, then

$$
\|u+e\| \leq \sum_{i}\left|u_{i}+e_{i}\right| \leq \sum_{i} r_{i 2} .
$$

Since $T_{2}<T$, Assumption (A4) ensures that

$$
\left\|x\left(T_{2}\right)\right\|<r .
$$

Writing down the solution of Eq. (7), and using Eq. (D.1), we have

$$
\begin{aligned}
\|x(t)\|=\left\|e^{A\left(t-T_{2}\right)} x\left(T_{2}\right)+\int_{T_{2}}^{t} e^{A(t-\tau)} B(u+e) d \tau\right\| \quad \forall t \geq T_{2} \\
\leq\left\|e^{A\left(t-T_{2}\right)} x\left(T_{2}\right)\right\|+\left\|\int_{T_{2}}^{t} e^{A(t-\tau)} B d \tau\right\| \sum_{i} r_{i 2}
\end{aligned}
$$

It is easy to verify that the first term is smaller than $\left(\bar{\lambda}_{p}\right.$ $\left./ \underline{\lambda}_{p}\right)^{1 / 2} r$, with the subscript $P$ denoting the solution of Eq. (4) and $r$ as given by Eq. (D.2), and the second term can be made smaller than any small positive number $\kappa$ since $A$ is a stable matrix and $r_{i 2}$ can be made arbitrarily small by Lemma 2 . Finally, we obtain

$$
\|x(t)\| \leq\left(\bar{\lambda}_{p} / \underline{\lambda}_{p}\right)^{1 / 2} r+\kappa=r_{p}
$$

Hence, $x(t) \in B r_{p}$ for all $t \geq T_{2}$. This concludes our proof. 